\title{
The Study on a New Immune Optimization Routing Model
}

\author{
Jun Qin, Jiang-qing Wang, and Zi-mao Li \\ College of ComputerScience, South-central University for \\ Nationalities, Wuhan,430074, China \\ dr.qinj@gmail.com, wjqing2000@yahoo.com.cn, lizm@sdu.edu.cn
}

\begin{abstract}
The integrated network (mixed with fixed network, cellular wireless network and ad hoc network etc.) would have different dynamic properties to the single-technique-based network, such as the movement of nodes and the change of delay of links. In this paper, we propose a new dynamic multicast routing model with a mechanism called local rearrangement to handle the changes in integrated network. Furthermore, D-IOA, an immune algorithm based on clone process, which employs gene library to improve the effectiveness of D-IOA to meet the real-time requirement in online multicast routing fields, is given to optimize the multicast sub-tree within the range of local rearrangement. The simulation results indicate that our algorithm balances three metrics better compared with other two famous dynamic multicast routing algorithms.
\end{abstract}

Keywords: Integrated Network, Dynamic Multicast Routing, Immune Algorithm, Gene Library.

\section{Introduction}

With the proliferation of wireless communication and individual communication, more and more various connecting techniques appear. It can be seen that future network will be an integration of various network techniques such as fixed network, cellular wireless network and Ad-hoc network, which is called "integrated network" in this paper. In order to guarantee the end-to-end Service of Quality (QoS), the seamless communication between users in the integrated network becomes a very challenging issue. Especially, with the importance of routing techniques for multicast connections being emphasized, how to conduct multicast routing in this kind of integrated network environment should be a key research issue.

Multicast now has been viewed as a very important facility in communicating networks with the popularity of multimedia applications such as radio, TV, ondemand video and teleconferences 1 . For dynamic multicast routing, there have been a few of research to do it such as DGA (Dynamic greedy algorithm), SP (Source rooted shortest path), DP(Dynamic Prim), LRA (Lagrangian-Relaxation Algorithm) and DCMTCD, etc 2] which are good inexpensive heuristics for the dynamic multicast routing. 
However, considering that there exist many connecting techniques in the integrated network as we mentioned before, there could be many different possible dynamics such as the node's move, the change of state of link etc. All those changes, sometimes even much, affect the multicast tree. However, current dynamic multicasting routing models are not considering these kinds of updates but only the membership change. Accordingly, those models aren't able to match the dynamics of the integrated network very well.

The rest of this paper is organized as follows. In section 2 , we will give a new dynamic multicast routing model suitable for the integrated network. In section 3, "local rearrangement" mechanism is introduced. In section 4, an immune algorithm is proposed to optimize the multicast sub-trees within the range of the local rearrangement and in Section 5 and the last section, some simulations and summaries are given.

\section{A New Dynamic Multicast Routing Model}

In order to simulate the dynamics of integrated network better, in this paper, besides membership updates, we will take another two dynamics: node movement and the delay of link changes into consideration. That is to say, we will focus on a new dynamic multicast routing model specific to integrated network. It is obvious that it is much more challenging than static multicast model. As the first step of our research plan about dynamic routing research in integrated network, we will simplify properly the situation discussed above. We will ignore the network topology change (the link appear and/or disappear) due to the movement of nodes.

Definition 1 (Multiple Dynamic Constrained Multicast Routing problem (MDCMR)). Given a undirected graph $G(V, E)$; a source node s, a set of initial destination nodes $M_{0}$, the initial delay of any link e Delayo $(e), a$ series of link delay variation $U=\left\{u_{0}, u_{1} \ldots u_{i}, ..\right\}$, where $u_{i}$ is a two-tuple ( $e_{i}$, Delay $\left._{i}\right), e_{i} \in E$, Delay $i$ is updated delay of $e_{i}$; a series of connection request $R=\left\{r_{0}, r_{1}, \ldots r_{i} \ldots\right\}$, where $r_{i}$ is a two-tuple $\left(y_{i}, o_{i}\right), y_{i} \in v, o_{i} \in\{j o i n$, leave $\}, a$ series of movement of node $H=\left\{h_{0}, h_{1}, \ldots, h_{g}\right\}$, where $h_{i}$ is a two-tuple (Old ${ }_{i}$, $\mathrm{New}_{i}$ ), Old $\mathrm{O}_{i}$ and $\mathrm{New}_{i}$ are old and new location of the moving node $i$.

As we can see, these three two-tuple series stand for link state change, multicast membership change and node movement, respectively. Supposing $M_{i}$ is the destination node set after suffering $u_{i}, h_{i}$ or $r_{i}$, The aim of MDCMR is to find a sequence of tree $\left\{T_{0}, T_{1}, \ldots T_{P}\right\}$ where each of them covers $S \cup M i$ and satisfies:

$$
\begin{gathered}
\text { Cost }\left(T_{i}\right)=\operatorname{Min} \sum_{e \in E_{T_{i}}} \cos t(e) \\
\text { Delay }(\operatorname{Path}(s, d))<\Delta, \forall d \in M_{i}
\end{gathered}
$$

The dynamics of MDCMR has three possibilities: the link change, the membership change and the movement of node. It is simple logically to analyse under which conditions the current multicast tree $T_{i-1}$, possibly need to be reconstructed . 


\section{Local Rearrangement Mechanism}

Before we discuss the routing strategy based on our new dynamic multicast routing model, there are two main principles should be considered carefully:

(1) Track the optimal or near-optimal multicast routing during the whole multicast session, which is described as the optimization model defined in this section.

(2) Reduce the extension and frequency of adaptation to current multicast tree. This is because if we adapt the current multicast tree dramatically and frequently, the current multicast session must be interrupted often, and hence it is very hard to guarantee the QoS.

In some sense, although we can consider MDCMR as a series of static multicast trees optimization problem, optimizing a multicast tree from scratch will be too costly time-consumed to satisfy the real- time update. Moreover, the QoS requirement of multicast sessions cannot tolerate frequent dramatic changes. Thus, to achieve this balance between cost, time and extension of changes of the tree, we will adopt an idea [3] of accumulating the impact to a tree and triggering a rearrangement based on a threshold represented by $Q F$ which is defined in literature 3 to measure the extension of changes to a multicast tree. Further, even when it's time to rearrange a multicast tree, we don't need to optimize the whole multicast tree from the scratch. Just the part of a tree affected directly by the changes is chosen to rearrange.

The details of how to decide the range of sub-tree and the related changes' handling are the similar to the process used in Ref [3].

\section{Optimization of Multicast Sub-tree}

As soon as the rearrangement of the sub-Tree is needed, an algorithm called D-IOA is used to reconstruct the sub-Tree and the newly generated sub-tree will be later combined with the left part of original multicast tree to form a complete multicast tree without loops.

Up to now, the problem in front of us has become a kind of static multicast tree construction problem within part of original multicast tree (in the range of sub-Tree). Here, we will utilize immune algorithm frame based on population search to solve the typical optimization problem. Based on our previous successful research in static multicast routing domain [4], still, the main component of D-IOA is clone process, a famous immune process in AIS, which is composed of two phrases: clone (same meaning as copy) and mutation.

But it is noticeable that our task is to compute online the multicast route and hence the real-time requirement is highlight considered by the optimization algorithms. Hence we have to give the revised version of IOA called dynamic IOA (D-IOA) to match the real-time feature. To achieve this, "gene library" will be introduced into our algorithm.

The details of D-IOA are given in Fig. 1. 
Input: A given network $G=(V, E)$, the state of network, a revised multicast session request $\left(s^{\prime}, M^{\prime}, \Delta^{\prime}\right)$

Output: An optimized multicast sub-trees $\left(s^{\prime}, M^{\prime}\right)$

1. Use the Dijkstra K-th shortest algorithm to construct the gene library, and delete the paths violating the delay constraints.

2. Initialize the population $P_{0}$, where $\left\lfloor d_{0} \% \cdot\left|P_{0}\right|\right\rfloor$ individuals produced from gene library and the remaining individuals by random construction. Those individuals violating the delay constraint should be deleted and re-produced again. $\mathbf{T}=\mathbf{0}$.

3. Delete the ring paths existed in individuals.

4. Compute the cost of each individual as the fitness $f_{i}$ of it.

5. Order the individuals based on $f_{i}$ of individuals in non-decreasing order. Suppose the rank of each individual is $\operatorname{Rank}_{i}, 1 \leq i \leq|P(t)|$.

6. For each individual $T_{i}$, compute $p_{i}=\frac{1 / f_{i}}{\sum_{j=1}^{|P(t)|} 1 / f_{j}}$, and then clone (copy) $\delta \cdot|P(t)| \cdot p_{i}$ individuals to produce clone population $\mathrm{Q}_{i}$.

7. Conduct mutation operator for each clone population $Q_{i}$ and then select the best multicast tree into $P(t+1)$

8. If the size of population ¿PopSize, delete $|P(t)|-$ PopSizeindividuals with biggest $f_{i}$ value.

9. $\mathrm{t}=\mathrm{t}+\mathbf{1}$.

10. If not satisfy the termination condition, goto 4 ; else goto 11 .

11. return the best multicast tree in population

Fig. 1. The pseudocode of D-IOA

In D-IOA, An individual means a multicast sub-tree. There are three important parameters: population size PopSize, the size of gene library $k$, the rate of initial individuals produced from gene library $d_{0} \%$.

Gene library is composed of good genes. A new antibody produced from gene library has good quality with much higher possibility than produced randomly. In gene library, there is more than one path from source node to destination nodes. Which are limited within the range of sub-tree. Dijsktra K-th shortest path algorithm is used to construct gene library. The details are that for each pair of source node and destination node, Dijkstra K-th shortest path algorithm is used to find $\mathrm{K}$ least-delay paths satisfying the delay constraint. The paths set for all pair nodes forms gene library and the size of it should be $\mathrm{K}^{*}\left|M^{\prime}\right|$.

\section{Experimental Results}

In this section, we present the results of the simulation experiments that were conducted to analyze the performance of our algorithm. We compared our algorithm with two other famous algorithms. We will first define the performance metrics, then describe the simulation model, and finally present and discuss the results. 


\subsection{Performance Metrics}

To analyze the performance of our algorithm, we used the following metrics:

(1) Cost Ratio $(C R)$ and Average Cost Ratio $(A C R)$. Those two metrics is a measure of how well a given algorithm performs (as regards cost of tree) in relation to the optimal algorithm. Since the online multicast problem is NPcomplete, it will be impractical to use the optimal algorithms as a basis for comparison. Hence, we use the BSMA heuristic proposed by Zhu [5] as our benchmark. Suppose that $T_{i}, 1 \leq i \leq n$ and $T_{i}^{\prime}, 1 \leq i \leq n$ are the sequences of trees respectively constructed by our algorithm and BSMA algorithm after undergoing $n$ changes in network. Then, the $\mathrm{CR}$ is given by the expression:C $R_{i}=$ $\frac{C\left(T_{i}\right)}{C\left(T_{i}^{\prime}\right)}$, where $C(T)$ is the cost of tree $T$. the $A C R$ is defined as: $A C R=\frac{\sum_{i=1}^{n} C R_{i}}{n}$.

$(2)$ Tree Change $(T C)$ and Average Tree Change $(A T C)$ : we define TC as follow: for a sequence of $\mathrm{n}$ update requests, let an algorithm produce the sequence of $T_{i}, 1 \leq i \leq n$, then TC is given by $T C_{i}=\left|\left(E\left(T_{i}\right)-E\left(T_{i+1}\right)\right) \cup\left(E\left(T_{i+1}\right)-E\left(T_{i}\right)\right)\right|$; and $A T C$ is defined as: $A T C=\frac{\sum_{i=1}^{n-1} T C_{i}}{n-1}$, where $E(T)$ denotes the set of edges in tree $T$ and $|\bullet|$ denotes the size of the set. A lower value of TC or ATC indicates that tested algorithm is better able to accommodate changes in the group without excessively modifying the tree.

(3) CPU Time $(C T)$ and Average CPU Time $(A C T)$. For our simulation purpose, $C T_{i}, 1 \leq i \leq n$ (or $A C T$ ) is defined as the (average) time taken by a single (or by sequential) update of network for the algorithm.

\subsection{Simulation Model}

To generate four kinds of possible changes in the network discussed above for our simulation, we have used the probabilistic model similar to the model employed in [3]. In a network of size $N$, let $k$ represents the number of member nodes in the multicast tree. Supposing the probabilities of an add-request, a remove-request, the movement of a multicast node and a delay change of a link are defined as the way in [3].

\subsection{Simulation Parameters and Simulation Results}

Our simulation studies were conducted on a set of 100 random networks. A random graph model proposed by Waxman [6] was used to produce the network. This ensures that the simulation results are independent of the characteristics of any particular network topology.

The size of gene library $k=10$. The dynamic change of those four types are chosen randomly with the probability expression above. The threshold parameter to trigger a rearrangement algorithm is $\rho=0.6$. In order to verify the performance of D-IOA, we compare our algorithm with DCMTCD [7] and LRA [8] algorithms. The main parameters used in D-IOA is $P_{0}=20, d_{0} \%=80 \%, \theta=0.4$. Every simulation result is the average of 50 running for tested algorithms. The 


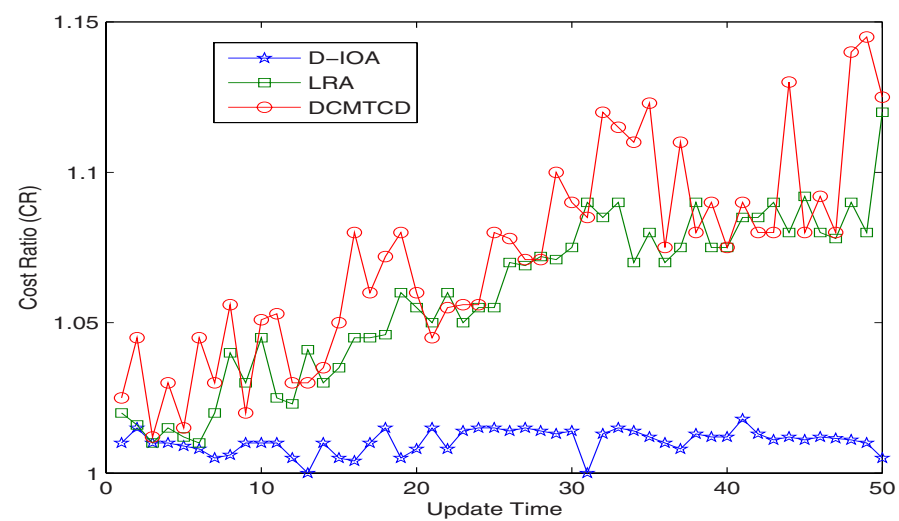

Fig. 2. the dynamic curve of CR

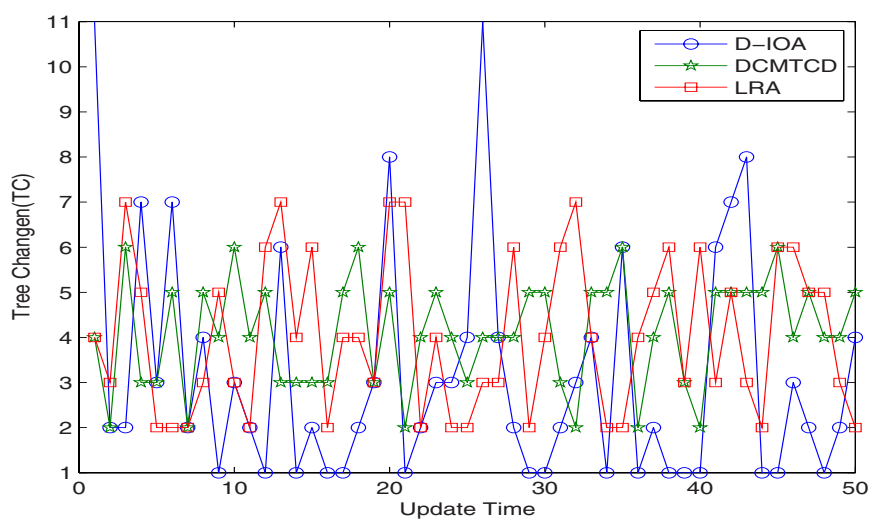

Fig. 3. the dynamic curve of TC

initial multicast group size is 20. Each simulation will be suffered 50 changes with only single change considered each time. All algorithms are implemented on a P4 2.4GHz machine with 256Mb memory. Fig.2, Fig.3 and Fig.4 shows respectively the $C R, T C, C T$ curves along with the 50 dynamic changes for the three algorithms. Table 1 shows the values of $A C R, A T C, A C T$.

As we can see from Fig. 2 and Table 1, the multicast tree found by D-IOA does not suffer an obvious cost increase along with the dynamic change of network while the DCMTCD and LRA do. The key different Between D-IOA and the latter two algorithms is that the latter two algorithms do not have the rearrangement mechanism, which means that rearrangement mechanism used in D-IOA indeed help a lot to improve the quality of multicast trees. From the Fig.3 and Table1, we can see that the average tree change (ATC) got by D-IOA is still lower than both the DCMTCD and LRA, although TC value become higher at 


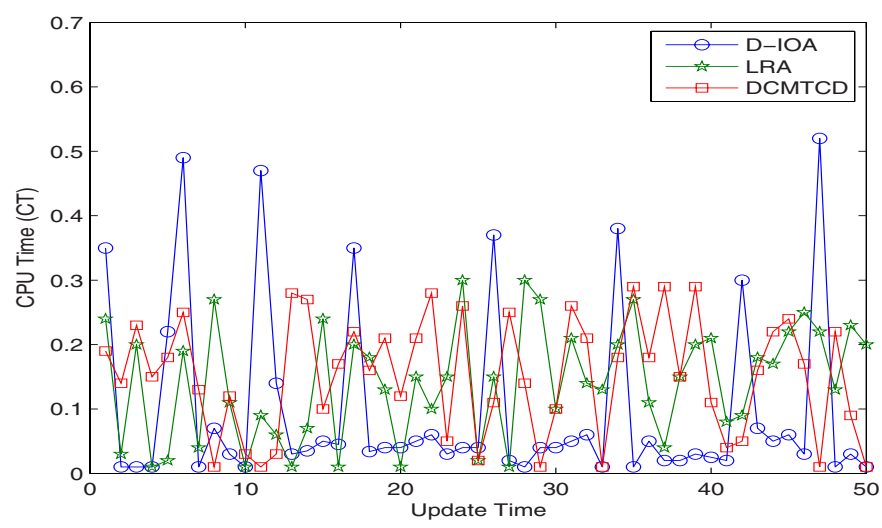

Fig. 4. The dynamic curve of CT

Table 1. The average performance of the three algorithms

\begin{tabular}{|l|l|l|l|}
\hline & ACR & ATC & ACT \\
\hline D-IOA & 1.013 & 3.7 & 0.087 \\
\hline LRA & 1.084 & 5.8 & 0.153 \\
\hline DCMTCD & 1.049 & 4.9 & 0.142 \\
\hline
\end{tabular}

the right time rearrangement triggered. Similarly, from Fig.4 and Table 1, we can see that the CT used by D-IOA is shorter than DCMTCD and LRA. Even though our algorithm introduces the rearrangement mechanism, we still have acceptable computation overhead because we limit the rearrangement to a local range rather than a whole multicast tree. Taking all the three aspects discussed here to be considered, our algorithm improves the performance of the dynamic multicast routing problem obviously.

\section{Summary}

In this paper, we proposed a new dynamic multicast routing model and a new algorithm to solve it. In this new model, we firstly considered another two dynamics ignored before in integrated network: the movement of node and the delay change of links. Both of them happen often in integrated network. In order to meet two important and contradicting goals: tracking the least-cost after each update and minimizing the frequent large changes to the multicast tree, we designed a local rearrangement mechanism and related algorithm to optimize the multicast sub-tree within local rearrangement range. The simulation results revealed that our algorithm balanced the two contradicting goals very well due to the introduction of local rearrangement mechanism in our algorithm. Also, the use of gene library further improves the effectiveness of rearrangement algorithm in that it makes best use of priori knowledge. 
Our further research will include: the sensitivity analysis of parameters in our algorithm; the convergence analysis of our algorithm; extensive version of our algorithm with multiple constraints such as delay jitter, bandwidth, packet loss, etc.

Acknowledgement. This study was supported by China NSF grant (No.60603008) and Hubei province PSF grant (No.2004ABA029).

\section{References}

1. Ravikumar, C.P., Bajpai, R.: Source-based delay-bounded multicasting in multimedia networks. Computer Communications 21 (1998) 126-132

2. Cobb, J.A.: Dynamic multicast trees. In: Networks, 1999. (ICON '99) Proceedings. IEEE International Conference on. (1999) 29-36

3. Raghavan, S., Manimaran, G., Siva Ram Murthy, C.: A rearrangeable algorithm for the construction of delay-constrained dynamic multicast trees. Networking, IEEE/ACM Transactions on 7(4) (1999) 514-529

4. Qin, J., Dong, W.Y., Chen, Y.P., Kang, L.S.: An immune-balance model and its preliminary application in apl problems. In Lishan, K., Zhihua, C., Xuesong, Y., eds.: Progress in Intelligence Computation \& Applications. (2005) 586-593

5. Hac, A., Zhou, K.L.: A new heuristic algorithm for finding minimum-cost multicast trees with bounded path delay. International Journal of Network Management 9(3) (1999) 265-278

6. Waxman, B.M.: Routing of multipoint connections. Selected Areas in Communications, IEEE Journal on 6(9) (1988) 1617-1622

7. Lin, H.C., Lai, S.C.: Vtdm-a dynamic multicast routing algorithm. Volume 3. (1998) 1426-1432 vol.3

8. Hong, S.P., Lee, H., Park, B.H.: An efficient multicast routing algorithm for delaysensitive applications with dynamic membership. In: INFOCOM '98. Seventeenth Annual Joint Conference of the IEEE Computer and Communications Societies. Proceedings. IEEE. Volume 3. (1998) 1433-1440 